\title{
IV. On a method of determining the specific gravity of small quantities of dense or porous bodies
}

\author{
J. Joly M.A.B.E.
}

To cite this article: J. Joly M.A.B.E. (1888) IV. On a method of determining the specific gravity of small quantities of dense or porous bodies, Philosophical Magazine Series 5, 26:158, 29-33, DOI: $10.1080 / 14786448808628232$

To link to this article: http://dx.doi.org/10.1080/14786448808628232

Published online: 29 Apr 2009.

Submit your article to this journal $₫$

Q View related articles $\longleftarrow$ 


\section{$\left[\begin{array}{ll}2 & 29\end{array}\right]$}

IV. On a Method of Determining the Specific Gravity of Small Quantities of Dense or Porous Bodies. By J. JoLy, H.A.B.E., Trinity College, Dublin*.

TT may happen to be of importance to the mineralogist or 1 to the chemist to determine the specific gravity of a very minute quantity of a solid. A method of effecting this, whatever the density of the solid or whatever its state of aggregation, is described in the following note. Jt, moreover, calls for the use of no special appliance. The method now in general use for the micro-determination of the specific gravities of silicates, \&c., of low density, is by balancing in a liquid of a specific gravity adjustable to that of the specimen, and subsequently determining the density of the solution employed.

This method fails altogether,

(1) when the substance has a specific gravity over four ;

(2) when the substance is of a porous nature.

In the first case the method fails for want of a liquid of sufficient density to equilibrate the solid. Be it observed that about 90 per cent. of the unsilicated mineral species range in density above $4: 5$.

In the second case - the case of porous bodies-the impossibility of freeing the body entirely from contained air, when immersed in liquids of the nature of those to which we are restricted, renders the method fallacious. There is another source of error. The particle in the first instance soaks in a certain quantity of liquid at such a density, subsequently the density of the liquid surrounding it is brought to another density. Is it assured that the density of the liquid lodged in the interstices of the substance in the first instance assumes the density subsequently conferred on the surrounding liquid? It might need a considerable time for the desired equalization of density to occur.

Briefly, the theory of the present method is as follows:The mineral by itself will not float in any known solution, suppose. If, however, we mix it with another substance of much lower specific gravity, there is easily found such a proportion for the constituents as will enable the mixed bodies to be equilibrated by dilution of the specific gravity liquid. We may, in short, adjust the specific gravity of the mixed substances to be as close to that of either of them as we please.

We require to know-

* Communicated by the Author. 
$W$ the weight of the mineral,

$\begin{array}{llll}\omega & \text { " } & \text { " sp. gr. } & \text { buoyant substance, } \\ s \quad \text { buoyant substance, }\end{array}$

in order to determine $\mathrm{S}$, the specific gravity required.

Then, as

$$
\begin{array}{r}
\text { sp. gr. }=\frac{\text { weight }}{\text { volume }}, \\
S=\frac{W}{\frac{W+\omega}{s}-\frac{\omega}{\sigma}}, \\
\text { or } \mathrm{S}=\frac{W \sigma s}{(W+\omega) \sigma-\omega s} .
\end{array}
$$

By this means, then, we can deal with bodies of any specific gravity ; and, further, if for the buoyant substance we chose one which, when brought to the liquid state, will creep into and surround the substance, we may evidently be independent of conditions of aggregation, and all trouble with contained air, or bubbles adhering to the surface of a rough fragment, avoided.

The method is practically carried out in the following manner :-

The specific gravity of a piece of translucent, homogeneous paraffin, free from bubbles, is taken by any of the ordinary methods-weighing in water with a sinker, or balancing in a mixture of alcohol and water, and then determining the density of the solution. The value found is what is called $\sigma$ above, the specific gravity of the buoyant substance. There is no better paraffin for our purpose than that sold in the form of candles. It fulfils all requirements.

From this piece of paraffin a little disk-shaped piece-about 3 or $4 \mathrm{~mm}$. in diameter, and $1.5 \mathrm{~mm}$. thick-is cut with a sharp knife, cleanly paired and smoothed on the edges by gently rubbing between the fingers. The disk is larger or smaller according to the quantity of mineral at our disposal, and if great accuracy be desired we determine its specific gravity, thus avoiding any assumption as to the homogeneousness of the piece from which it is cut. There will be in general, however, no need for so doing: thus, compare the two following specific gravities obtained-(1) on a piece of paraffin weighing over 11 grms.; (2) on a jittle disk removed from this, and weighing about 04 gram. (2) was determined by balancing in dilute alcohol :-

$$
\text { (1) } 0.9204
$$

-an inappreciable difference of specific gravity. The large piece may be preserved for future determinations. 
The disk removed is next weighed in a delicate balance. If as small as described above, the balance should read definitely to $0.2 \mathrm{mgr}$. Its weight is $\omega$ in the equation. It is in all cases manipulated by use of a clean ivory forceps. If very minute it is weighed on a tared watch-glass, and so need not be manipulated at all after preparation. Removed from the balance, the small fragment (or fragments) of mineral is placed upon the surface of the disk. The extremity of a slip of copper, about $5 \mathrm{~mm}$. wide, is now heated in a smokeless flame- it is better to use a little copper ball, drilled and fitted on to a fine steel knitting-needle-and held above the fragment of mineral, care being taken not to approach it so closely as to endanger the paraffin being volatilized or of its being melted so far as to risk loss by running over. Preferably the disk of paraffin should rest on a piece of clean copper; this will keep the lower surface cool. In point of fact, the mineral in general absorbing heat more freely than the paraffin, melts the paraffin beneath it by conductivity, and there is little risk of loss. The heating is continued till the mineral is seen to be completely soaked with the paraffin-every crack and cranny is then filled, the paraffin welling up and swallowing the specimen and expelling all trace of air.

When cold it is placed in the balance and weighed. By subtracting $\omega$ from the weight found, we have $W$, the weight of the mineral.

There is probably no loss of paraffin in this process. Thus it will be found that if such a pellet be very carefully balanced in a solution, removed, dried, and melted on the hitherto unaltered face of the disk, and then replaced in the solution, there is, if anything, a slight decrease of density ; on complete cooling this decrease is inappreciable.

The pellet is now dropped into a specific gravity solution. A saturated solution of common salt and water (sp. gr. about $1 \cdot 2$ ) will in many cases be found sufficient to float it. If so, we have merely to adjust by adding water. Otherwise we resort to Thoulet's solution (Minéralogie Micrographique, Fouqué et Lévy, p. 118). I have prepared no pellets approaching this density $(2 \cdot 77)$, but I prefer the use of this solution in all cases; it seems to concentrate less rapidly by evaporation, and is more "creepy."

In this operation of balancing it is advisable to use a camel's-hair brush for stirring, and also for conveying small quantities of liquid when finally adjusting-a process of much delicacy. The brush is also used for removing bubbles from the pellet, which, however, will be found to give little trouble if the solutions be previously boiled to expel air. 
If the mixed solutions containing the pellet be left standing for some hours before finally adjusting, it will be found on examination with a lens that bubbles will no longer gather on the paraffin.

The last operation is finding the specific gravity of this solution, which gives us $s$ in the formula. This is most accurately done in a Sprengel tube, holding about 2 cc.; the bottle may also be used.

The table opposite contains the results of some determinations made by the method.

Of experiments 4 and 5 it is interesting, perhaps, to note that 4 was undertaken with the notion that the mineral being dealt with was barite. Its weight, as a band specimen, was deceptive, it being penetrated by sphalerite. On getting the result $(2 \cdot 78)$ it was concluded that an oversight had been made somewhere in the measurements, and experiment 5 was undertaken; this giving $2 \cdot 77$, the specimen was appealed to. Tests then showed it to be calcite. The determinations numbered 11 and 12 were effected on minute, greenish crystals, héxagonal in shape, removed from the Wicklow granite. The crystals alone sank rapidly in Thoulet's solution (sp. gr. 2.77). The foregoing method was resorted to, and the specific gravity of apatite being obtained, the substance was tested in the usual way. The tests being confirmatory, it was concluded that the mineral was apatite, a rare substance in this granite.

I have thought well to include in the table some of the quantities obtained in working the formula, as bearing on the scale on which the experiments have been made. It is evident that the method can be applied on a smaller scale still ; weighing to tenths of milligrams, 10 milligrams conld be dealt with. The method, it is seen, involves two weighings and one determination of specific gravity by balancing in a solution; that is, if a piece of paraffin of known specific gravity be at hand.

Recently it has come to my notice that a method based on a similar principle is described in Rosenbusch's Mikroskopische Physiographie der Mineralien und Gesteine. In detail, however, the methods differ essentially. Thus in Rosenbusch's method the mineral is caused to adhere to a piece of wax by pressure, and is subsequently taken off and weighed after their joint specific gravity is determined. Here the difficulty of freeing the substance from contained or adhering air remains. A porous substance cannot be dealt with. They differ, too, in other respects, so that I am induced to think the foregoing may still, independently, be of use. 


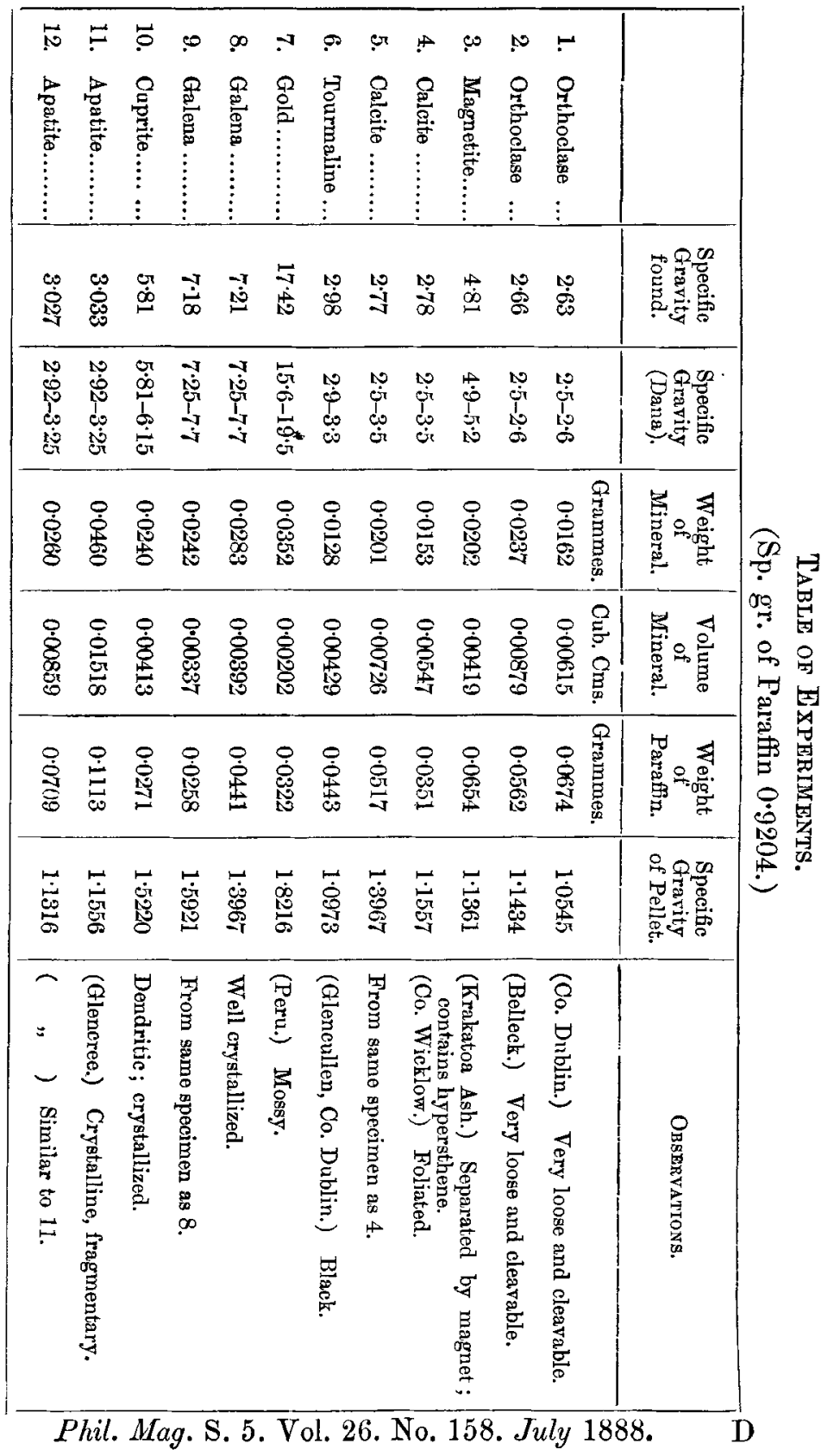

